\title{
Assessment of the BD MGIT TBc Identification Test for the Detection of Mycobacterium tuberculosis Complex in a Network of Mycobacteriology Laboratories
}

\author{
Diana Machado, ${ }^{1}$ Jorge Ramos, ${ }^{1}$ Isabel Couto,,${ }^{1,2}$ Nureisha Cadir, ${ }^{3}$ Inácio Narciso, ${ }^{3}$ \\ Elizabeth Coelho, ${ }^{4}$ Sofia Viegas, ${ }^{3}$ and Miguel Viveiros ${ }^{1,5}$ \\ ${ }^{1}$ Grupo de Micobactérias, Unidade de Microbiologia Médica, Instituto de Higiene e Medicina Tropical, \\ Universidade Nova de Lisboa (IHMT/UNL), Rua da Junqueira 100, 1349-008 Lisboa, Portugal \\ ${ }^{2}$ Centro de Recursos Microbiológicos (CREM), UNL, 2829-516 Caparica, Portugal \\ ${ }^{3}$ Instituto Nacional de Saúde, Ministério da Saúde de Moçambique, 264 Cidade de Maputo, Mozambique \\ ${ }^{4}$ Programa Nacional de Controlo da Tuberculose, Ministério da Saúde de Moçambique, 264 Cidade de Maputo, Mozambique \\ ${ }^{5}$ Centro de Malária e Outras Doenças Tropicais/LA, IHMT/UNL, Rua da Junqueira 100, 1349-008 Lisboa, Portugal
}

Correspondence should be addressed to Miguel Viveiros; mviveiros@ihmt.unl.pt

Received 11 October 2013; Accepted 18 November 2013; Published 23 January 2014

Academic Editor: Tomasz Jagielski

Copyright (C) 2014 Diana Machado et al. This is an open access article distributed under the Creative Commons Attribution License, which permits unrestricted use, distribution, and reproduction in any medium, provided the original work is properly cited.

We evaluate the performance of the TBcID assay in a panel of 100 acid-fast bacilli cultures. Sixty-four isolates were TBcID positive for Mycobacterium tuberculosis complex (MTBC), whereas 36 gave negative results. These included 28 nontuberculous mycobacteria, one nonmycobacterial isolate, one M. tuberculosis, and six M. bovis BCG strains. This corresponds to a sensitivity of $90.14 \%$, specificity of $100 \%$, and positive and negative predictive values of $100 \%$ and $80.55 \%$, respectively. The test is rapid, easy to perform and interpret, and does not require sample preparation or instrumentation. However, a negative result does not exclude the presence of a strain belonging to MTBC, especially when mutations in $m p b 64$ gene are present or some M. bovis BCG strains are isolated. The TBcID showed potential to assist in the identification of MTBC when the implementation and usage of molecular methods are often not possible, principally in resource-limited countries.

\section{Introduction}

The genus Mycobacterium comprises over 150 species of which more than 30 can cause disease in livestock, wildlife, and humans $[1,2]$. Members of the Mycobacterium tuberculosis complex are the etiologic agents of tuberculosis and the responsible for about 8.6 million of new tuberculosis cases and 1.3 million deaths in 2012 [3]. Nontuberculous mycobacteria are a cause of opportunistic infections and are frequently encountered in clinical samples [1]. In some cases, clinical presentation of pulmonary disease caused by nontuberculous mycobacteria is very similar to that of tuberculosis [4]. Therefore, rapid diagnosis of patients with active tuberculosis is of major importance for the control of the disease demanding for reliable, easy to perform instrument-free identification assays, especially for low-income countries. Fast and accurate differentiation between $M$. tuberculosis complex and nontuberculous mycobacteria is essential, as it enables the implementation of appropriate measures to prevent the spread of the infection, allows the implementation of appropriate therapy, and prevents inappropriate drug susceptibility testing without species identification. Moreover, the differentiation of nontuberculous mycobacteria is important, since some of these species are resistant to most of the first line antibiotics used in tuberculosis therapy [4].

The conventional methods employed for the diagnosis of mycobacterial diseases rely on acid fast staining, culture, and phenotypic characterization. The development and implementation of liquid culture media allowed the reduction of the time for detection of positive cultures. From these, the BACTEC MGIT 960 is the most sensitive for recovery of mycobacteria from clinical samples [5]. After the positivity 
of a culture, the verification of the presence or absence of acid fast bacilli in the culture can be achieved by means of microscopy. Nevertheless, it does not distinguish between $M$. tuberculosis and nontuberculous mycobacteria. Usually, most laboratories in resource-limited settings use the labor intensive standard biochemical tests to identify $M$. tuberculosis complex, which requires subculture of mycobacteria on solid media and delay the result by several weeks. This process increases the turnaround time for reporting positive results. Nucleic acid probe and amplification based methods have been used for the identification of mycobacteria from cultures or directly from clinical samples thus reducing the time for diagnosis [6,7]. However, since molecular methods do not distinguish between live and death bacteria, culture confirmation is mandatory. Moreover, these techniques require specific equipment, expensive reagents requiring refrigeration, and highly trained personnel.

Lateral flow assays, also called immunochromatographic assays, have been developed for the discrimination between $M$. tuberculosis complex and nontuberculous mycobacteria. These include the SD Bioline Ag MPT64 Rapid assay (Standard Diagnostics, Kyonggi-do, Korea), Capilia TB (TAUNS, Numazu, Japan), and the MGIT TBc Identification Test (Becton Dickinson Diagnostic Instrument Systems, Sparks, MD). These sandwich-type assays use a monoclonal antibody to detect the MPB64 protein (Rv1980c; also termed as MPT64), which is specifically secreted during growth by the $M$. tuberculosis complex [8]. The MPB64 is a $24 \mathrm{kDa}$ protein, highly specific for the $M$. tuberculosis complex, except some variants of Mycobacterium bovis BCG $[9,10]$. In this study we investigated the performance of the BD MGIT TBc identification test for the discrimination between M. tuberculosis complex and nontuberculous mycobacteria grown in both liquid and solid medium for the routine discrimination of $M$. tuberculosis complex in our setting and the reliability of the MPB64 protein for $M$. tuberculosis complex identification. This assay is intended to be used as a preliminary screening identification test when the hospital laboratories only perform acid-fast smear staining and culture and send their isolates to intermediate or reference laboratories for molecular identification at the species level and susceptibility testing of M. tuberculosis.

This study was carried out as part of the training programs in TB laboratory diagnosis for Mozambique and other Portuguese speaking countries, created and implemented to assess the usefulness of this assays for routine identification of M. tuberculosis in the network of hospital mycobacteriology laboratories in these countries as well as in Portugal.

\section{Methods}

2.1. Mycobacterial Strains and Culture. A total of 100 culture isolates received from seven hospitals of the Lisbon Health Region during the training period of three months were included in this study. These comprised 71 strains of the $M$. tuberculosis complex, 28 nontuberculous mycobacteria, and one acid-fast bacilli other than mycobacteria (Table 1). $M$. tuberculosis $\mathrm{H} 37 \mathrm{Rv}$ and M. bovis BCG Pasteur strain were used as controls. As an intermediate level laboratory in the network of hospital mycobacteriology laboratories in Lisbon,
TABLE 1: Identification of $M$. tuberculosis complex and differentiation from nontuberculous mycobacteria by the TBcID.

\begin{tabular}{lcc}
\hline \multirow{2}{*}{ Mycobacterial species $(n)$} & \multicolumn{2}{c}{ TBcID } \\
& Positive & Negative \\
\hline M. tuberculosis complex (71) & 64 & 7 \\
Nontuberculous mycobacteria (28) & 0 & 28 \\
AFB high G+C bacteria (1) & 0 & 1 \\
\hline
\end{tabular}

AFB: acid-fast bacilli.

the Mycobacteriology Laboratory of the IHMT/UNL only receives acid-fast smear positive cultures for molecular identification and drug susceptibility testing from the hospital laboratories. All isolates were processed for Ziehl-Neelsen staining and inoculated into MGIT culture tubes and Lowenstein-Jensen slants. Samples were digested and decontaminated, by the standard sodium hydroxide-N-acetyl-Lcysteine (NaLC-NaOH) method [11]. Once a positive signal was given by the BACTEC system, a Ziehl-Neelsen staining was performed and the presence or absence of serpentine cording morphology was observed. Only acid-fast bacilli positive cultures were included in the study. Presence of contamination was evaluated by inoculation of the cultures into blood agar plates and Ziehl-Neelsen staining.

2.2. TBcID Assay. The TBcID assay (Becton Dickinson Diagnostic Instrument Systems, Sparks, MD) consists of a nitrocellulose membrane on a test device with immobilized antiMPB64 mouse monoclonal antibodies conjugated with gold colloidal for the detection of the MPB64 protein. In the presence of a sample, the antibody-colloidal gold conjugate binds to the MPB64 antigen and flows laterally through the membrane until the reaction zone. Here, the complex will be captured by a second antibody specific for MPB64. If the MPB64 protein is present, a purple band will be developed. Each TBcID device was inoculated with $100 \mu \mathrm{L}$ of a positive MGIT culture. All cultures were tested with growth units above 100 (GU $\geq 100)$ and between day 1 and day 5 after positivity within the MGIT system. Selected M. tuberculosis isolates from the same collection were evaluated with cultures grown in Lowenstein-Jensen. For that, one loopful of colonies was suspended in $200 \mu \mathrm{L}$ of extraction buffer (phosphate buffer with $0.05 \%$ Tween 20 and $0.02 \%$ sodium azide) and $100 \mu \mathrm{L}$ of the suspension used in the assay. The results were interpreted $15 \mathrm{~min}$ after application of the sample. A positive result was indicated by the development of two purple bands, one in the control zone $(\mathrm{C})$ and another in the test zone $(\mathrm{T})$. The presence of the control band alone indicates a negative result.

\subsection{Confirmatory Identification Tests. All the results obtained} with TBcID were compared with Accuprobe MTBC culture identification test (GenProbe Inc., San Diego, CA) as the "gold standard" for the identification of the M. tuberculosis complex. Briefly, $1 \mathrm{~mL}$ of the each culture was centrifuged during $10 \mathrm{~min}$ at $13000 \mathrm{rpm}$ and the pellet was used for hybridization according to the instructions of the manufacturer. The isolates that were negative for M. tuberculosis 
TABLE 2: Comparison of the results obtained by the TBcID and the Accuprobe assay.

\begin{tabular}{|c|c|c|c|c|}
\hline \multirow{2}{*}{ Mycobacterial species $(n)$} & \multicolumn{2}{|c|}{ TBcID } & \multicolumn{2}{|c|}{ Accuprobe } \\
\hline & Positive $(n)$ & Negative $(n)$ & Positive $(n)$ & Negative $(n)$ \\
\hline \multicolumn{5}{|l|}{ M. tuberculosis complex } \\
\hline M. tuberculosis (64) & 63 & 1 & 64 & 0 \\
\hline M. bovis BCG (6) & 0 & 6 & 6 & 0 \\
\hline M. africanum (1) & 1 & 0 & 1 & 0 \\
\hline \multicolumn{5}{|l|}{ Nontuberculous mycobacteria } \\
\hline M. abscessus (2) & 0 & 2 & 0 & 2 \\
\hline M. avium (4) & 0 & 4 & 0 & 4 \\
\hline M. intracellulare (5) & 0 & 5 & 0 & 5 \\
\hline M. chelonae (2) & 0 & 2 & 0 & 2 \\
\hline M. fortuitum (1) & 0 & 1 & 0 & 1 \\
\hline M. genavense (2) & 0 & 2 & 0 & 2 \\
\hline M. gordonae (4) & 0 & 4 & 0 & 4 \\
\hline M. kansasii (1) & 0 & 1 & 0 & 1 \\
\hline M. marinum (2) & 0 & 2 & 0 & 2 \\
\hline M. peregrinum (1) & 0 & 1 & 0 & 1 \\
\hline M. scrofulaceum (1) & 0 & 1 & 0 & 1 \\
\hline M. szulgai (1) & 0 & 1 & 0 & 1 \\
\hline M. xenopi (1) & 0 & 1 & 0 & 1 \\
\hline M. ulcerans (1) & 0 & 1 & 0 & 1 \\
\hline \multicolumn{5}{|l|}{ Nonmycobacteria } \\
\hline AFB high $\mathrm{G}+\mathrm{C}$ bacteria (1) & 0 & 1 & 0 & 1 \\
\hline
\end{tabular}

complex by Accuprobe were identified using the Genotype CM/AS (Hain, Nehren, Germany) according to manufacturer's instructions. Total genomic DNA for the Genotype CM/AS assays was extracted from the cultures using the QIAamp DNA mini kit (QIAGEN, GmbH, Hilden, Germany) according to the manufacturer's instructions.

2.4. Assessment of Discordant Results. The isolates that were positive for M. tuberculosis complex with Accuprobe but negative with the TBcID were tested using the Genotype MTBC (Hain) assay, according to manufacturer's instructions. Mutations in the $m p b 64$ gene of $M$. tuberculosis were analyzed by PCR amplification and DNA sequencing using the primers $m p b 64$-F30 and mpb64-R433, mpb64-F404 and mpb64-R891, described elsewhere [12]. The reaction mixtures were prepared for a total reaction volume of $50 \mu \mathrm{L}$ consisting of $1 \mathrm{x}$ Taq buffer (Fermentas, Ontario, Canada), $1.5 \mathrm{mM} \mathrm{MgCl}_{2}$, $200 \mathrm{mM}$ of each dNTP, $10 \mathrm{pmol}$ of each primer, $1.5 \mathrm{U}$ Taq DNA Polymerase (Fermentas), and $5 \mu \mathrm{L}$ of chromosomal DNA. The PCR reactions were performed with the following amplification profile: initial denaturation at $94^{\circ} \mathrm{C}$ for $5 \mathrm{~min}$, followed by denaturation at $94^{\circ} \mathrm{C}$ for $1 \mathrm{~min}$, annealing at $62^{\circ} \mathrm{C}$ for $1 \mathrm{~min}$, and extension at $72^{\circ} \mathrm{C}$ for $1 \mathrm{~min}$ during 40 cycles. The final extension occurred at $72^{\circ} \mathrm{C}$ for $10 \mathrm{~min}$. PCR products were sequenced with an $\mathrm{ABI}$ Prism 3130 capillary sequencer (Applied Biosystems, Foster City, CA) and the BigDye terminator kit (ABI Prism).

2.5. Performance Analysis. The sensitivity, specificity, and positive and negative predictive values of the TBCID assay were determined using the results of the Accuprobe MTBC culture identification test as the gold standard.

\section{Results}

The results obtained are summarized in Tables 1 and 2 . Of the 100 mycobacterial cultures tested, 64 were correctly identified as $M$. tuberculosis complex by the TBcID. The assay failed to detect one $M$. tuberculosis isolate that carried a mutation in the mpt64 gene and all M. bovis BCG tested $(n=6)$. We did not observe cross-reaction with any of the 28 nontuberculous mycobacteria and the one nonmycobacterial acid-fast bacilli culture tested. These correspond, in this study, to a sensitivity of $90.14 \%$, specificity of $100 \%$, positive predictive value of $100 \%$, and negative predictive value of $80.55 \%$ of the TBcID assay for the identification of the M. tuberculosis complex.

From the seven false negatives yielded by TBcID one corresponded to an $M$. tuberculosis strain as determined by Accuprobe. To evaluate if the negative result was due to a reduced amount of secreted MPB64 protein necessary for the detection, this isolate was subcultured and the test was repeated. The result was again negative. After this, the entire mpb64 gene was sequenced and an insertion of two bases (CG) at position 335 of the gene was detected. This frame shift resulted in the generation of a premature stop codon at amino acid position 167, truncating the protein. The remaining six isolates were identified as $M$. bovis BCG by the Genotype MTBC assay and these isolates were later on found to belong to one child BCGitis and from patients that are being monitored for bladder cancer immunotherapy [13]. Testing by 


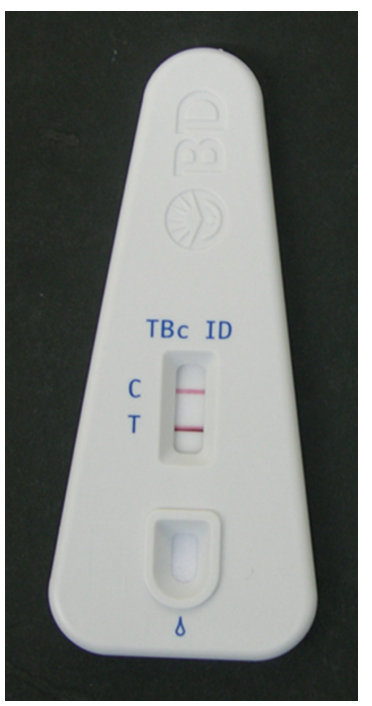

(a)

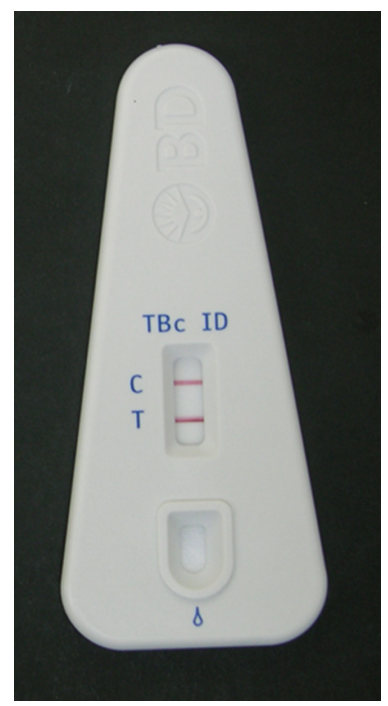

(b)
FIGURE 1: Identification of $M$. tuberculosis complex isolates by the MGIT TBC ID assay from (a) positive MGIT culture and (b) Lowenstein-Jensen slants. In the Figure it is shown the result of the TBCID for the same isolate when grown in different culture media. The positive result is indicated by the development of two purple bands, one in the control zone (C) and another in the test zone $(\mathrm{T})$.

polymerase chain reaction with the four primers confirmed that the mpb64 gene is absent in these strains.

The manufacturer's instructions of the TBcID system recommend its use with isolates grown in liquid media only. Nevertheless, we decided to test the capability of the test to detect MPB64 protein in strains grown on solid media. For that we selected $25 \mathrm{M}$. tuberculosis isolates from the panel of isolates grown in liquid media previously evaluated. The test demonstrates a good performance for detection of $M$. tuberculosis complex from solid cultures as all isolates were correctly identified as $M$. tuberculosis complex (Figure 1 and Table 3).

\section{Discussion}

In this work, the performance of the BD MGIT TBc identification test was evaluated for the identification of $M$. tuberculosis complex and differentiation from nontuberculous mycobacteria. We did not detect false positive results. However, the TBcID assay yielded seven false negative results. The assay demonstrates $100 \%$ of specificity which is similar to that published in other studies [14-16]. Nevertheless, the sensitivity was found to be $90.14 \%$, which is lower than that reported by other authors (between 95.2 and 100\%) [14-16]. This fact can be explained by the false negative results obtained.

The occurrence of false negatives can be due to the absence of the MPB64 protein or mutations in the coding gene, $m p b 64$. Several studies have reported false negatives results for several BCG strains $[14,17,18]$. Among the $M$. bovis BCG variants, some of them do not produce the MPB64
TABLE 3: Results of the TBcID for a subset of 25 M. tuberculosis complex strains using different culture media.

\begin{tabular}{lcc}
\hline \multirow{2}{*}{ Strain ID } & \multicolumn{2}{c}{ TBcID result } \\
& MGIT & Lowenstein-Jensen \\
\hline 22 & $(+)$ & $(+)$ \\
28 & $(+)$ & $(+)$ \\
56 & $(+)$ & $(+)$ \\
57 & $(+)$ & $(+)$ \\
58 & $(+)$ & $(+)$ \\
61 & $(+)$ & $(+)$ \\
70 & $(+)$ & $(+)$ \\
74 & $(+)$ & $(+)$ \\
75 & $(+)$ & $(+)$ \\
76 & $(+)$ & $(+)$ \\
77 & $(+)$ & $(+)$ \\
78 & $(+)$ & $(+)$ \\
79 & $(+)$ & $(+)$ \\
80 & $(+)$ & $(+)$ \\
81 & $(+)$ & $(+)$ \\
87 & $(+)$ & $(+)$ \\
90 & $(+)$ & $(+)$ \\
91 & $(+)$ & $(+)$ \\
94 & $(+)$ & $(+)$ \\
95 & $(+)$ & $(+)$ \\
96 & $(+)$ & $(+)$ \\
97 & $(+)$ & $(+)$ \\
98 & $(+)$ & $(+)$ \\
99 & $(+)$ & $(+)$ \\
100 & $(+)$ & $(+)$ \\
\hline Total (25) & 25 \\
\hline 5 & 25 &
\end{tabular}

$(+)$ positive result.

antigen, whereas others are good secretors of this antigen [19]. This difference is due to the deletion of the mpb64 gene together with the RD2 [20]. One of the strains included in this study was the BCG Pasteur, already described as a nonproducer of the MPB64 antigen [21, 22]. In Portugal, BCG SSI (strain 1331) is used for vaccination and BCG Medac is used for treatment of noninvasive urothelial bladder carcinoma and thus we assume that we have found both strains in our study, since one strain came from a child who developed osteomyelitis after BCG vaccination and five strains were isolated from patients undergoing cancer therapy [13]. Another possible explanation for the occurrence of negative results is the presence of mutations within the mpb64 gene. Several mutations are reported in the literature for this gene $[12,15,16$, $18,22-24]$. In this study, we detected a GC insertion at nt 335 , which resulted in a truncated protein and a TBCID negative result for this $M$. tuberculosis strain. As far as we know, this mutation has not been previously reported. Further, due to the existence of some strains of $M$. tuberculosis with delayed MPB64 secretion some cultures might lead to erroneous reporting of negative results. Vadwai et al. [25] propose that a culture must be tested with $\mathrm{GU} \geq 300$ to avoid false negative results. In our study, we did not detect false negative results using as cutoff MGIT cultures with GU $\geq 100$. 
Differentiating $M$. tuberculosis complex from nontuberculous mycobacteria as soon as possible is important, mainly in situations in which nontuberculous mycobacteria strains represent a considerable portion of mycobacteria isolated [26]. The nontuberculous mycobacteria and one acid fast bacilli other than mycobacteria tested in this study correctly provided true negative results. Serpentine cord morphology can be used for rapid presumptive identification of $M$. tuberculosis in liquid culture and as a guide for the selection of auxiliary tests. Using this feature, we were able to improve the sensitivity of the test since the combination of the serpentine cording morphology with the TBcID comparing with AccuProbe assay as the gold standard corresponds to a sensitivity and specificity of $100 \%$ and identical positive and negative predictive values (data not shown). Similar results were reported by others [27]. The capacity of the TBcID was also evaluated for the identification of a subset of $25 \mathrm{M}$. tuberculosis strains grown on solid media, and all the 25 isolates were correctly identified as M. tuberculosis complex.

The main advantages of the test are the cost and rapidity. However, it has some disadvantages. The method (i) cannot be applied directly to clinical samples; (ii) does not allow the identification at species level; and (iii) requires further confirmatory tests for identification at species level. The MPB64 protein is the target of the test but it is also its weak point. All negative results suspected for $M$. tuberculosis complex had to be tested with other methodologies. Noteworthy, the isolation of $M$. bovis from human samples is uncommon, but sporadically, BCG strains are isolated from patients receiving BCG immunotherapy. The fact that all of our BCG strains are negative for MPTB64 can be useful to assist in the detection of BCG strains since it can be used as a screening tool in combination with molecular methods. Our results show that the TBcID is not an alternative to the Accuprobe system, at least in our work algorithm, due to the false negative results presented and this also precludes its use for confirmatory laboratory diagnosis of tuberculosis infections. Another limitation is related to the safety measures. The test necessitates biosafety level 3 conditions to be applied as it involves a considerable bacterial inoculum. When these conditions are not present, the test must be performed with heat-inactivated cultures [14].

The BD MGIT TBc identification test showed potential to assist in the identification of $M$. tuberculosis complex and differentiation from nontuberculous mycobacteria when the implementation and usage of molecular methods are often not possible, principally in resource-limited countries. The test is simple, rapid, easy to perform and interpret, and does not require sample preparation or instrumentation. In Mozambique, in 2012 the TB Reference Laboratories from Maputo and Beira have introduced the immunochromatographic assay, a very important step in a country for which very limited information regarding the occurrence of nontuberculous mycobacteria is available [28]. In 2012, among all positive cultures, $20.5 \%$ were from single or mixed infection from nontuberculous mycobacteria, a strong evidence of the high prevalence of nontuberculous mycobacteria in this country that are often misidentified and might be considered as multidrug resistant tuberculosis.
This study demonstrated the usefulness of the immunochromatographic assays for routine identification of $M$. tuberculosis in a network of mycobacteriology laboratories as preliminary screening identification test of cultures to be sent to the intermediate or reference laboratory as part of the network of TB laboratories of the national tuberculosis control programs.

\section{Conflict of Interests}

The authors declare that there is no conflict of interests regarding the publication of this paper.

\section{Acknowledgments}

The authors wish to thank the collaborating hospitals for their kind cooperation. The authors wish to thank Dr. Teresa Pacheco for the help with the BCG vaccine strain. This work was supported by grants "Projecto de Formação em Diagnóstico da Tuberculose (ForDILAB-TB)” from Fundação Calouste Gulbenkian (FCG, Portugal) and the Community of the Portuguese Speaking Countries (CPLP), and the project "A implementação de um novo método de identificação rápida do complexo M. tuberculosis nos Labs de Referência da Tuberculose de Maputo e Beira: a sua importância para a validação dos testes de susceptibilidade aos tuberculostáticos" from FCG. D. Machado was supported by Grant no. SFRH/BD/65060/2009 from Fundação para a Ciência e a Tecnologia (FCT, Portugal).

\section{References}

[1] J. O. Falkinham III, "Surrounded by mycobacteria: nontuberculous mycobacteria in the human environment," Journal of Applied Microbiology, vol. 107, no. 2, pp. 356-367, 2009.

[2] J. Stanford and C. Stanford, "Mycobacteria and their world," International Journal of Mycobacteriology, vol. 1, no. 1, pp. 3-12, 2012.

[3] World Health Organization, "Global tuberculosis control: WHO report 2013,” Tech. Rep. WHO/HTM/TB/2013.11, World Health Organization, Geneva, Switzerland, 2013.

[4] D. E. Griffith, T. Aksamit, B. A. Brown-Elliott et al., "An official ATS/IDSA statement: diagnosis, treatment, and prevention of nontuberculous mycobacterial diseases," American Journal of Respiratory and Critical Care Medicine, vol. 175, no. 4, pp. 367416, 2007.

[5] E. Tortoli, P. Cichero, C. Piersimoni, M. T. Simonetti, G. Gesu, and D. Nista, "Use of BACTEC MGIT 960 for recovery of mycobacteria from clinical specimens: multicenter study," Journal of Clinical Microbiology, vol. 37, no. 11, pp. 3578-3582, 1999.

[6] D. F. Moore, J. A. Guzman, and L. T. Mikhail, "Reduction in turnaround time for laboratory diagnosis of pulmonary tuberculosis by routine use of a nucleic acid amplification test," Diagnostic Microbiology and Infectious Disease, vol. 52, no. 3, pp. 247254, 2005.

[7] I. K. Neonakis, Z. Gitti, E. Krambovitis, and D. A. Spandidos, "Molecular diagnostic tools in mycobacteriology," Journal of Microbiological Methods, vol. 75, no. 1, pp. 1-11, 2008.

[8] P. Andersen, D. Askgaard, L. Ljungqvist, J. Bennedsen, and I. Heron, "Proteins released from Mycobacterium tuberculosis 
during growth," Infection and Immunity, vol. 59, no. 6, pp. 19051910, 1991.

[9] M. Harboe, S. Nagai, M. E. Patarroyo, M. L. Torres, C. Ramirez, and N. Cruz, "Properties of proteins MPB64, MPB70, and MPB80 of Mycobacterium bovis BCG," Infection and Immunity, vol. 52, no. 1, pp. 293-302, 1986.

[10] R. Yamaguchi, K. Matsuo, A. Yamazaki et al., "Cloning and characterization of the gene for immunogenic protein MPB64 of Mycobacterium bovis BCG," Infection and Immunity, vol. 57, no. 1, pp. 283-288, 1989.

[11] P. T. Kent and G. P. Kubica, Mycobacteriology: A Guide for the Level III Laboratory, US Dept of Health and Human Services, Public Health Service, Centers for Disease Control, Atlanta, Ga, USA, 1985.

[12] D. Hillemann, S. Rüsch-Gerdes, and E. Richter, "Application of the Capilia TB assay for culture confirmation of Mycobacterium tuberculosis complex isolates," The International Journal of Tuberculosis and Lung Disease, vol. 9, no. 12, pp. 1409-1411, 2005.

[13] L. Lima, J. A. Ferreira, A. Tavares et al., "FASL polymorphism is associated with response to bacillus Calmette-Guérin immunotherapy in bladder cancer," Urologic Oncology: Seminars and Original Investigations, vol. 32, no. 1, pp. 1-44, 2014.

[14] T. Gaillard, M. Fabre, C. Martinaud, R. Vong, P. Brisou, and C. Soler, "Assessment of the SD Bioline Ag MPT64 Rapid and the MGIT TBc identification tests for the diagnosis of tuberculosis," Diagnostic Microbiology and Infectious Disease, vol. 70, no. 1, pp. 154-156, 2011.

[15] P.-L. Lu, Y.-C. Yang, S. C. Huang et al., "Evaluation of the bactec MGIT 960 system in combination with the MGIT TBc identification test for detection of Mycobacterium tuberculosis complex in respiratory specimens," Journal of Clinical Microbiology, vol. 49, no. 6, pp. 2290-2292, 2011.

[16] A. Martin, D. Bombeeck, K. Fissette et al., "Evaluation of the BD MGIT TBc Identification Test (TBc ID), a rapid chromatographic immunoassay for the detection of Mycobacterium tuberculosis complex from liquid culture," Journal of Microbiological Methods, vol. 84, no. 2, pp. 255-257, 2011.

[17] N. Hasegawa, T. Miura, K. Ishii et al., "New simple and rapid test for culture confirmation of Mycobacterium tuberculosis complex: a multicenter study," Journal of Clinical Microbiology, vol. 40, no. 3, pp. 908-912, 2002.

[18] M.-C. Yu, H.-Y. Chen, M.-H. Wu et al., "Evaluation of the rapid MGIT TBc identification test for culture confirmation of Mycobacterium tuberculosis complex strain detection," Journal of Clinical Microbiology, vol. 49, no. 3, pp. 802-807, 2011.

[19] M. A. Behr and P. M. Small, "A historical and molecular phylogeny of BCG strains,” Vaccine, vol. 17, no. 7-8, pp. 915-922, 1999.

[20] G. G. Mahairas, P. J. Sabo, M. J. Hickey, D. C. Singh, and C. K. Stover, "Molecular analysis of genetic differences between Mycobacterium bovis BCG and virulent M. bovis," Journal of Bacteriology, vol. 178, no. 5, pp. 1274-1282, 1996.

[21] C. Abe, K. Hirano, and T. Tomiyama, "Simple and rapid identification of the Mycobacterium tuberculosis complex by immunochromatographic assay using anti-MPB64 monoclonal antibodies," Journal of Clinical Microbiology, vol. 37, no. 11, pp. 36933697, 1999.

[22] K. Hirano, A. Aono, M. Takahashi, and C. Abe, "Mutations including IS6110 insertion in the gene encoding the MPB64 protein of Capilia TB-Negative Mycobacterium tuberculosis isolates," Journal of Clinical Microbiology, vol. 42, no. 1, pp. 390392, 2004.
[23] M. Muyoyeta, P. E. W. de Haas, D. H. Mueller et al., "Evaluation of the Capilia TB assay for culture confirmation of Mycobacterium tuberculosis infections in Zambia and South Africa," Journal of Clinical Microbiology, vol. 48, no. 10, pp. 3773-3775, 2010.

[24] K. Ngamlert, C. Sinthuwattanawibool, K. D. McCarthy et al., "Diagnostic performance and costs of capilia TB for Mycobacterium tuberculosis complex identification from broth-based culture in Bangkok, Thailand," Tropical Medicine and International Health, vol. 14, no. 7, pp. 748-753, 2009.

[25] V. Vadwai, M. Sadani, R. Sable et al., "Immunochromatographic assays for detection of Mycobacterium tuberculosis: what is the perfect time test?" Diagnostic Microbiology and Infectious Disease, vol. 74, no. 3, pp. 282-287, 2012.

[26] I. Couto, D. Machado, M. Viveiros, L. Rodrigues, and L. Amaral, "Identification of nontuberculous mycobacteria in clinical samples using molecular methods: a 3-year study," Clinical Microbiology and Infection, vol. 16, no. 8, pp. 1161-1164, 2010.

[27] G.-H. Shen, C.-H. Chen, C.-H. Hung et al., "Combining the Capilia TB assay with smear morphology for the identification of Mycobacterium tuberculosis complex," International Journal of Tuberculosis and Lung Disease, vol. 13, no. 3, pp. 371-376, 2009.

[28] INSIDA, Ministry of Health of Mozambique, Mozambique's National Statistics Institute, and ICF Macro, National Survey on Prevalence, Behavioral Risks and Information about HIV and AIDS in Mozambique, 2009, Calverton, Md, USA, 2010. 

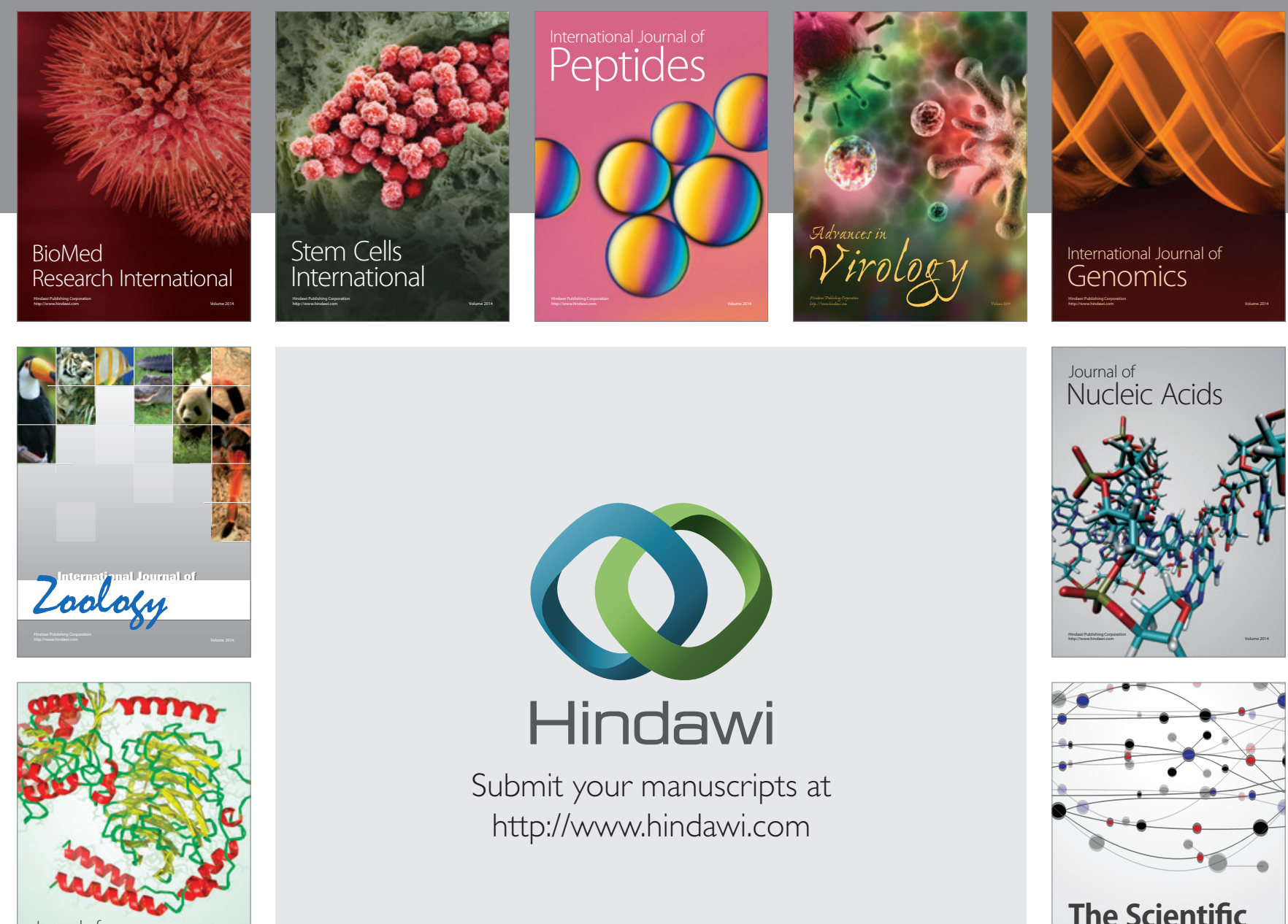

Submit your manuscripts at

http://www.hindawi.com

Journal of
Signal Transduction
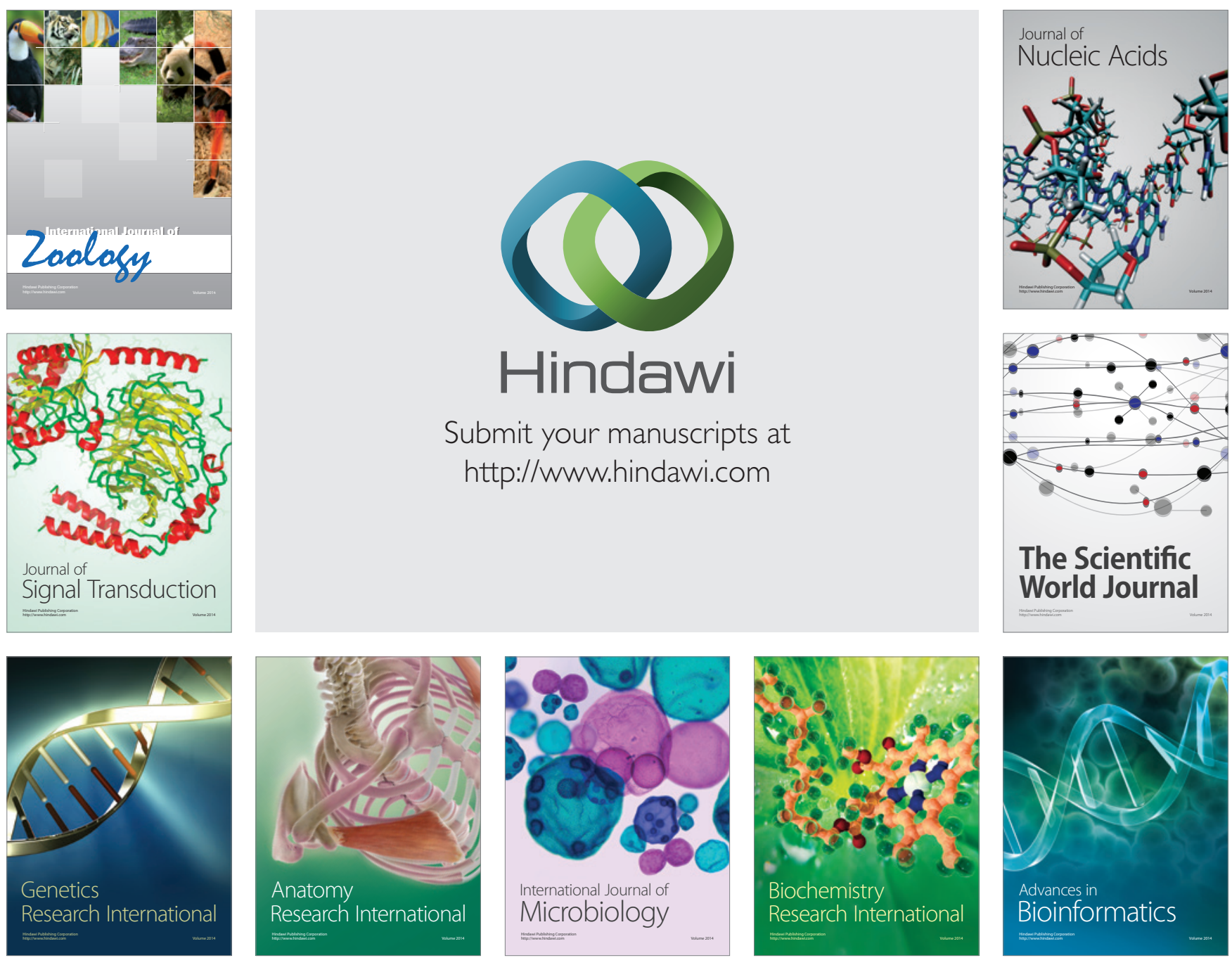

The Scientific World Journal
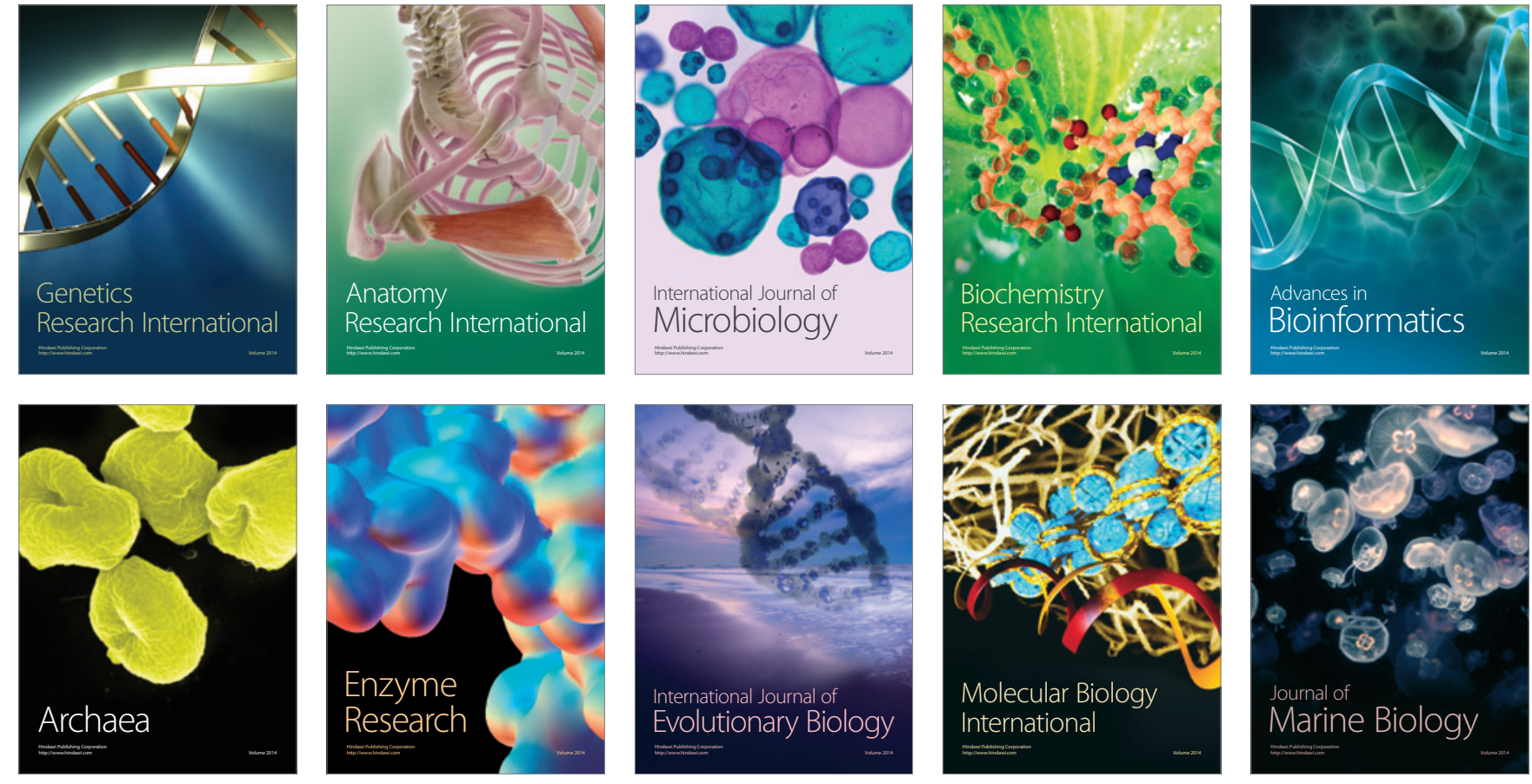